\title{
Fault Location Modeling and System Design of Aircraft Cable
}

\author{
Chengcheng WANG ${ }^{\mathrm{a}, 1}$, Wenlong $\mathrm{YU}^{\mathrm{b}}$, Xin WANG ${ }^{\mathrm{a}}$, Chu-wan XIAO ${ }^{\mathrm{a}}$, Longjie \\ ZHANG $^{\mathrm{a}}$ \\ a Naval Aeronautics University, China \\ ${ }^{b}$ Unit 91213
}

\begin{abstract}
In view of the fact that aircraft has frequent line faults, which seriously threatens flight safety and lacks effective means of line fault detection, based on the analysis of traditional cable fault detection and location methods, a more suitable method for fault detection and location of aircraft cables is explored. On the basis of theoretical research, further develops of the software and hardware design of cable fault detection and location system is presented based on improved spread spectrum time domain reflectometry. The testing method and flow chart are put forward and applied to actual aircraft cable testing. The influence of different test conditions on test precision is verified by test experiments of different types of cables. It provides a theoretical reference for aircraft cable fault detection and location, and provides a design idea for further solving the actual aircraft line test requirements.
\end{abstract}

Keywords. Fault location, time domain reflectometry, improved spread spectrum time domain reflectometry, aircraft cable of different materials.

\section{Introduction}

In recent years, aircraft line faults occur frequently, which seriously threatens flight safety. The demand for aircraft line fault detection is becoming more and more urgent. Common types of cable faults: open circuit faults, low resistance (short circuit) faults, high resistance faults, intermittent faults, mixed faults, etc [1-2]. Cable aging, wear, arc ignition and many other reasons can lead to cable failure, and cable failure is likely to cause catastrophic consequences for aircraft [3]. In the actual aircraft structure, most of the aircraft cables are in multi-segment, multi-junction connection state, which is difficult to be detected by the same equipment at the same time [4]. Deformation and serious attenuation bring great errors to fault diagnosis. Traditional cable fault detection methods can not meet the needs of aircraft cable testing.

Based on the above problems, this paper aims at the need of aviation cable fault detection and location, and carries out single-end detection of open fault and short-circuit Fault in aviation cable based on improved spread spectrum time domain reflectometry (SSTDR) technology. When the test signal is injected from one end of the cable, the reflection signal will be generated when the impedance mismatch point is encountered, by accurately measuring the time difference between the reflected signal and the

${ }^{1}$ Corresponding Author, Chengcheng WANG, Naval Aeronautics University, NO.188 Second Road, Yantai, Shandong, China; E-mail: 497990518@qq.com. 
transmitted signal and combining the propagation speed, the distance between the fault point and the test starting point can be obtained, the influence of different conditions on the test accuracy of different types of cable is verified by test experiments.

\section{Analysis of Fault Location Method for Aircraft Cable}

\subsection{Comparison and Analysis of Cable Fault Location Methods}

First paragraph.At present, the common test methods for cable fault detection and location are bridge method, high voltage pulse method, second pulse method, third pulse method, time domain pulse reflection method and so on [5].

The bridge method is the most classical cable fault testing method [6] Its basic principle is to use the bridge balance principle. The bridges used are resistance bridge, capacitance bridge, low voltage bridge, high voltage bridge and so on. The instrument based on bridge test principle has many advantages such as simple circuit implementation, easy cost control and so on. However, when the bridge method is used to detect cable faults, both ends of the cable must be operated at the same time, which leads to the disadvantage that it can not be used in aircraft cable fault detection.

High-voltage pulse method [7] is widely used in fault detection of power cables, but aviation cables are usually low-voltage cables, which can not withstand thousands of volts of high voltage. Usually, the high-voltage pulse method is a blind area when the test distance is less than $10 \mathrm{~m}$. The length of cables on airplanes varies from several meters to tens of meters, so high-voltage pulse method can not be used.

In the second pulse method [8], the low voltage pulse is first emitted, and the waveform of the unbroken cable is obtained. The waveform is stored as a reference waveform. For high resistance faults, the waveform is as good as that of the cable. The waveform of this method is simple and easy to identify. However, the aviation cable can not withstand the high voltage that can be withstood on the power cable, so this method is not suitable for aviation cable detection.

Time Domain Pulse Reflectometry (TDR) [9] has come to maturity after many years of development. The fault type is determined by observing the reflected pulse waveform, and the fault distance is determined by measuring the time interval between the transmitted pulse and the reflected pulse.. It has the advantages of high accuracy, no damage to cable and small measurement blind area. It is suitable for aviation cable fault detection. In recent years, Sequential time domain reflectometry (STDR) and spread spectrum time domain reflectometry (SSTDR) [10]. are extended to realize on-line detection, which further improves the accuracy and anti-interference of detection and location. The following are detailed analysis of these methods.

\subsection{Time Domain Reflection Method}

Time domain reflection method is also called pulse reflection method or radar method. The voltage pulse is sent to the communication cable, and the obstacle point is determined by using the principle that the time difference between the transmitted pulse and the reflected pulse of the fault point is proportional to the distance of the fault point.

The basic theory of time domain reflection method is the transmission line theory. When the signal is transmitted in the cable, it will reflect at the fault point where the wave impedance changes. The incident wave voltage of the cable is $U_{i}$, the wave 
impedance of the normal cable is $Z_{1}$, the fault equivalent wave impedance is $Z_{2}$, the signal propagates from $Z_{1}$ to $Z_{2}$, the reflected voltage wave is $U_{f}$, and the voltage reflection coefficient is: ' $\beta_{\mathrm{u}}=\frac{\mathrm{Z}_{2}-\mathrm{Z}_{1}}{\mathrm{Z}_{2}+\mathrm{Z}_{1}}=\frac{\mathrm{U}_{\mathrm{f}}}{\mathrm{U}_{\mathrm{i}}}$.

When pulse measurement is used, the distance between the fault point and the transmitting point and the fault type are determined according to the time when the reflected wave arrives at the transmitting point and the waveform characteristics of the reflected wave.

(1) When the transmitting wave encounters an open-circuit fault, that is, $Z_{2} \rightarrow \infty$, $\beta_{\mathrm{u}}=1$, the polarity of the reflected wave and the incident wave is the same.

(2) When the transmitting wave encounters a circuit-breaking fault, i.e. $Z_{2} \rightarrow 0$, $\beta_{\mathrm{u}}=-1$, the polarity of the reflected wave is opposite to that of the incident wave.

(3) When the transmitting wave encounters low resistance fault, $\mathrm{Z}_{2}<\mathrm{Z}_{1}, \beta_{\mathrm{u}}<0$, the polarity of reflected wave $\mathrm{U}_{\mathrm{f}}$ and incident wave $\mathrm{U}_{\mathrm{i}}$ is opposite, and $\left|\mathrm{U}_{\mathrm{i}}\right|<\left|\mathrm{U}_{\mathrm{f}}\right|$.

(4) When the transmitting wave encounters high resistance fault, $\mathrm{Z}_{2} \approx \mathrm{Z}_{1}, \beta_{\mathrm{u}}=0$, and there is no reflection wave. If the distance from the fault point to the test end is $\mathrm{L}$, then: $\mathrm{L}=\frac{\mathrm{V}_{\mathrm{p}} * \mathrm{t}}{2}$.

It can be seen that pulse reflection method depends on the obvious change of impedance of obstacle points, and can not detect high resistance and intermittent faults of cables, and is not suitable for poor insulation obstacles with larger resistance values. Because the transmitted pulse signal has an impact on communication signals, pulse method can not realize on-line detection. For the single cable off-line short circuit, open circuit and other hard faults, the test reflection peak is obvious, and it can be used for off-line hard fault testing.

\subsection{Spread Spectrum Time Domain Reflection Method}

The test signal emitted by SSTDR method is generated by pseudo-random sequence (PN code) modulated with sinusoidal wave of the same frequency. It has high immunity to noise and good ability of cross-joint. The high anti-noise performance of the test signal shows its superiority in fault diagnosis of aircraft cables.

Before the PN sequence is transmitted, BPSK is needed to modulate the sequence signal. When the modulated signal is injected into the cable, the amplitude of the transmitted signal can be set very small and there is no interference to the working signal in the cable (figure 1).

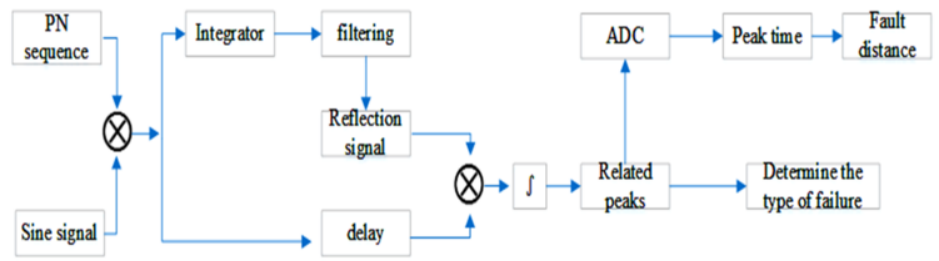

Figure 1. A schematic diagram of SSTDR cable fault detection

The purpose of modulating PN codes is to improve the signal-to-noise ratio when correlating. The system can identify useful signals more easily, immunize the signals against interference, and make the correlation peak of signals more obvious, which can 
improve the ability of interference and noise filtering. The method of SSTDR is superior to STDR in anti-interference and measurement accuracy.

\section{Design of Aircraft Cable Fault Location System}

According to the theoretical analysis of cable fault detection and location, the aircraft cable fault detection and location system is mainly composed of power supply module, signal processing module, signal conditioning module, multi-meter measurement module, display, keyboard and so on.

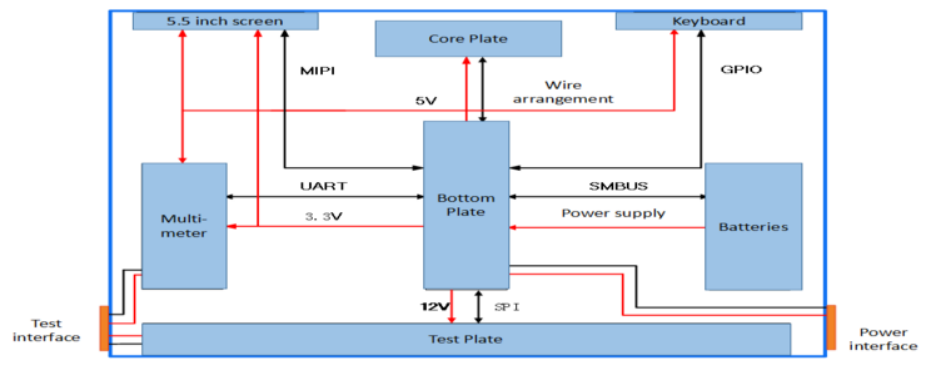

Figure 2. Hardware composition of cable fault location analyzer

The design framework of aviation cable fault detection system is shown in figure 2. Users input test control information through keyboard display. After reading the control information of interface, the device generates the specified transmitting signal and outputs it. The transmitting signal reaches the cable under test through the conditioning circuit and reflects at the fault. The test plate receives the leaking signal (co-transmitting signal) leaked by the conditioning circuit and the cable fault. Finally, the incident signal is correlated with the signal received by $\mathrm{AD}$ to get the fault information.

\section{Fault Detection and Result Query}

\subsection{Operation Flow of Fault Detection}

The operation flow of the fault detection interface is as follows. The main interface is equivalent to the desktop of the ordinary computer. It is an interface that automatically enters after booting. As shown in figure 3, the functions of the interface display are as follows:

- Fault detection;

- Voltage measurement;

- Resistance measurement;

- Micro-resistance measurement;

- Set up;

- Data management. 


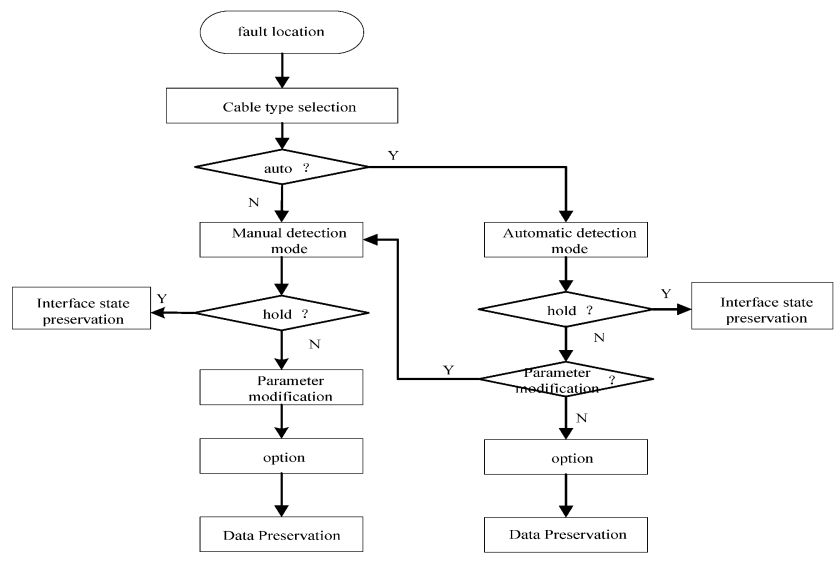

Figure 3. Fault detection flow chart

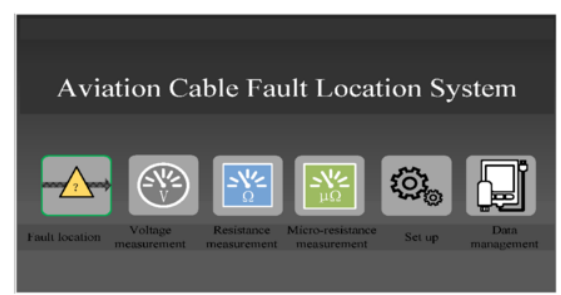

Figure 4. Main interface of cable fault locator

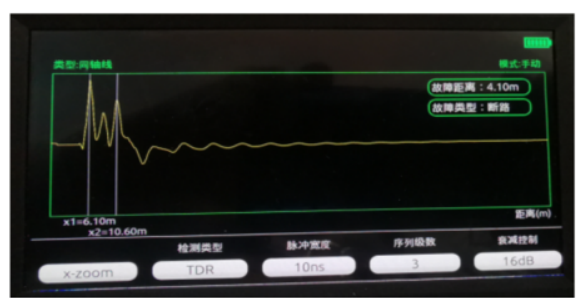

Figure 5. Fault detection interface

After entering the main interface, the default selection of the system is "fault detection", which can switch other functions through knob control, or directly enter its corresponding functional interface through digital keys; the top of the main interface can display time and power (figure 4 and figure 5).

\subsection{Experimental Analysis of Factors Affecting Test Precision}

It should be noted that the test accuracy of different types of cable is restricted by many factors, such as transmitting signal type, correlation algorithm, and so on. For single-core shielded cable, twisted-pair Cable and 1553B bus, three types of aerial cable are tested under the same length $(10 \mathrm{~m})$, modulation type, sampling rate and related algorithms, the SSTDR method is used to study the influence of different PN chip length on the test results. The chip length of the original PN code to 7 and 15, test the three kinds of cable, and record the data (table 1).

Table 1. The results of the experiment

\begin{tabular}{|c|c|c|c|c|}
\hline NO. & Chip width & Sequence length & Type of cable & Fault signal reflection time \\
\hline 1 & $50(\mathrm{~ns})$ & 7 & Single-core shielding wire & $116(\mathrm{~ns})$ \\
\hline 2 & $50(\mathrm{~ns})$ & 15 & Single-core shielding wire & $114(\mathrm{~ns})$ \\
\hline 3 & $50(\mathrm{~ns})$ & 7 & Twisted pair & $95(\mathrm{~ns})$ \\
\hline 4 & $50(\mathrm{~ns})$ & 15 & Twisted pair & $99(\mathrm{~ns})$ \\
\hline 5 & $50(\mathrm{~ns})$ & 7 & $1553 \mathrm{~B}$ bus & $99(\mathrm{~ns})$ \\
\hline 6 & $50(\mathrm{~ns})$ & 15 & $1553 \mathrm{~B}$ bus & $100(\mathrm{~ns})$ \\
\hline
\end{tabular}


Analysis of experimental results: For a given cable, the longer the sequence length, the better the correlation waveform. For a $10 \mathrm{~m}$ cable, when the sequence length is 7 , the correlation waveform is perfect and the precision is good enough. if the continuous transmit chip width and length affect the test range, the influence formula is:

$$
\frac{1}{2} \times T_{c} \times v \leq L \leq \frac{1}{2} \times N \times T_{c} \times v
$$

Where the length is selected in conjunction with the chip width.

\section{Conclusion}

Based on the analysis of the traditional cable fault detection and location methods, this paper discusses the fault detection and location methods which are more suitable for the connecting state of aircraft cables, and compares and analyses their respective testing systems and advantages and disadvantages. Combining with the method analysis, the software and hardware design of the aircraft cable fault detection and location system based on TDR/STDR/SSTDR is further developed. The testing method and flow chart are put forward and applied to the actual aircraft cable test. The influence of different test conditions on test precision is verified by test experiments of different types of cables. The test result also checks out that the test accuracy of different types of cable is restricted by many factors. This puts forward certain technical requirement to the user. It provides a theoretical reference for the aircraft cable fault detection and location, and it also provides a reference for the aircraft cable fault detection and determination.

\section{References}

[1] Jeon, Jeong-Chay, Kim.Fault Detection of Low Voltage Cable using Time-Frequency Correlation in SSTDR[J].The Transactions of The Korean Institute of Electrical Engineers. 2015,64(3):498-504.

[2] Chen Wei, Wangli. Research on on-line fault detection and location technology of aviation cable[J] . Aircraft Design, 2010, 30(2): 62-67

[3] Mao Bodybuilding, Wang Li, Yang Shanshui. Research on online diagnosis method of multi-conductor cable fault in aircraft power system[J] . Journal of Instruments, 2016, 37(9): 1962-1970.

[4] Michael Ballas, Nicholas Locken, Charm Parkey, Craig Hughes. A Non-Destructive High-Voltage LowEnergy Intermittent Fault Location System[J]. AUTOTESTCON, IEEE. 2011:78-86.

[5] Geon Seok Lee, Gu-Young Kwon, Su Sik Bang, et al.Monitoring Electrical and Thermal Characteristics of HTS Cable Systems via Time-Frequency Domain Reflectometry[J]. IEEE Transactions on Applied Superconductivity, 2017,27(4):1-5.

[6] Shirkoohi Gholamhossein. Modeling of fault detection in electrical wiring[J].IET Science, Measurement \& Te Chay, Kim.Detection and Location of Cable Fault Using Improved SSTDR[J].The Transactions of The Korean Institute of Electrical Engineers. 2016,65(9):1583-1589.

[7] Wang Xin, Wang Wangjianshun, Zhoutao. Research on power cable fault location and detection signal denoising[J] .. Computer simulation. 2015,32(11): 137-141.

[8] Jianglin, Guoting, Hewenrong, etc.. Study on fault location of cable based on wavelet packet analysis[J] .. Smart Grid, 2015, 3(1): 39-42.

[9] Diyuyao, Guogang.. New method of data de-noising and fault location for aeronautical cable TDR[J] . Modern Defence Technology, 2016, 44(6): 128-134.

[10] Lilele, Jiang Yang, Liuyuhong. SSTDR based cable fault detection algorithm[J]. Electronic Technology, 2015, 8(2): 81-83. 